\title{
THEOBALDIA (CULICELLA) LITOREA (Shute 1928) ET ANOPHELES (ANOPHELES) ALGERIENSIS Théobald 1903, DANS L'OUEST DE LA FRANCE. CONTRIBUTION A L'ÉTUde ÉCOLOGIQUe DE CES DEUX ESPÈces
}

\author{
Par J.-M. DOBY, B. RAULT et F. DAVID
}

En Europe, Theobaldia (Culicella) litorea (Shute 1928) et Anopheles algeriensis Theobald 1903, ont été, à plusieurs reprises, trouvés dans des stations relativement septentrionales, en Grande-Bretagne et en Allemagne du Nord. Ces stations sont estimées aberrantes ou accidentelles par Rioux (1958) : "L'Angleterre représente pour Anopheles (A.) algeriensis une station aberrante... », "...Nous pensons que cette espèce (Th. litorea), de caractère méridional, possède en Angleterre une localité aberrante...». Pour cet auteur, ces deux espèces sont «typiquement méditerranéennes ».

Les récoltes, en relative abondance, de ces deux espèces sur la côte Ouest de la France, de Bordeaux à Lorient, au cours des dernières années, faisant le pont entre les stations britanniques et les stations méditerranéennes, semblent bien infirmer l'opinion de Rioux. Si ces deux Culicidés n'ont, jusqu'à ces derniers temps, jamais encore été observés en France, en dehors des limites du bassin méditerranéen, il apparaît que c'est sans doute faute d'avoir été recherchés.

Pour ces deux espèces, nous ajoutons, dans l'Ouest de la France, les stations nouvelles suivantes :

Theobaldia litorea :

- Loire-Atlantique : nombreux gittes larvaires sur le pourtour de la Grande-Brière, au Nord de St-Nazaire.

- Morbihan : nombreux gites larvaires sur la côte, depuis le Golfe du Morbihan jusqu’à la limite du Finistère, à l'Ouest de Lorient.

- Charente-Maritime: plusieurs gites larvaires aux environs de Marennes. 
Nous donnons ci-dessous les stations antérieurement connues en Europe occidentale et en Afrique du Nord :

\section{Theobaldia litorea}

\begin{tabular}{|c|c|c|}
\hline Pays & Localité & Auteurs \\
\hline Gde-Bretagne . & $\begin{array}{l}\text { Essex (Walton-on-Naze) } \\
\text { Hampshire (Haylind Island) } \\
\text { Kent (Sandwich) } \\
\text { Suffolk (Cambridge) } \\
\qquad \text { id. }\end{array}$ & $\begin{array}{l}\text { Shute } 1928 \text { et } 1933 \\
\text { Marshall et Staley } 1933 \\
\qquad i d . \\
\text { in Marshall et Staley } \\
1933 \\
\text { Macan } 1933 \text { et } 1939 .\end{array}$ \\
\hline Irlande.... & Environs de Belfast & $\begin{array}{l}\text { in Marshall et Staley } \\
1933\end{array}$ \\
\hline France.... & $\begin{array}{l}\text { Camargue } \\
\text { Pyrénées-Orientales (Banyuls) } \\
\begin{array}{l}\text { Provence } \\
\text { Roussillon } \\
\text { Languedoc } \\
\text { Morbihan (Belle-Ile-en-Mer) }\end{array}\end{array}$ & $\begin{array}{l}\text { Harant et Rioux } 1954 \\
\text { Doby } 1955 \\
\text { Rioux } 1958 \\
\text { Doby et coll. } 1958\end{array}$ \\
\hline Espagne.... & Province du Cuenca & Torres-Cañamares 1945 \\
\hline Italie $\ldots \ldots \ldots$ & $\begin{array}{c}\text { Sardaigne (côte N.W., Uta) } \\
-\quad \text { (côte S., Alghero) }\end{array}$ & Aitken 1955 \\
\hline Algérie.... . & $\begin{array}{l}\text { Alger } \\
\text { Lac Halloula } \\
\text { Gué de Constantine }\end{array}$ & $\begin{array}{l}\text { Senevet et Andarelli } \\
\quad 1956\end{array}$ \\
\hline
\end{tabular}

Anopheles algeriensis :

- Morbihan : Couregan (à l'Ouest de Lorient) (une nymphe, un adulte).

- Charente-Maritime : Brouages et Hiers (plusieurs gites larvaires, nombreuses larves et nymphes).

Royan (un adulte cherchant à piquer sous abri). 


\section{Anopheles algeriensis}

\begin{tabular}{|c|c|c|}
\hline Pays & Localité & Auteurs \\
\hline Allemagne.... & Mecklembourg (Crefeld) & Martini (1) 1931 \\
\hline Gde-Bretagne . & $\begin{array}{l}\text { Norfolk. Diverses localités } \\
\text { Localité ?? }\end{array}$ & $\begin{array}{l}\text { Edwards } 1932 \\
\text { Marshall } 1938 \\
\text { Mattingly in Senevet et } \\
\text { Andarelli } 1956 \\
\text { Hart } 1954 \\
\text { Shute (2) } 1933\end{array}$ \\
\hline France.... & $\begin{array}{l}\text { Corse (Cattaraggio) } \\
-\quad \text { (Vico) } \\
-\quad \text { (dans basses terres) } \\
-\quad \text { (dans basses terres) } \\
\text { Provence } \\
\text { Roussillon (Font Estramer) } \\
\text { Charente-Marit. (La Rochelle) } \\
\text { Loire-Atlantique (environs de } \\
\text { St-Nazaire) }\end{array}$ & $\begin{array}{l}\text { Brumpt } 1925 \\
\text { Catanei } 1925 \\
\text { Jaujou et coll. } 1950 \\
\text { Jaujou et coll. } 1951 \\
\text { Toumanoff } 1954 \\
\text { Sautet } 1953 \\
\text { Sicart } 1956 \\
\text { Hedeen } 1957 \\
\text { Hedeen } 1957\end{array}$ \\
\hline Italie $\ldots \ldots \ldots$ & Sardaigne (diverses localités) & Aitken 1955 \\
\hline $\begin{array}{l}\text { Afrique } d u \\
\text { Nord } \ldots \ldots\end{array}$ & Diverses localités & $\begin{array}{l}\text { in Senevet et Andarelli } \\
1956\end{array}$ \\
\hline
\end{tabular}

Un certain nombre de gites larvaires ont été l'objet d'une étude écologique. Les résultats en sont donnés dans le tableau ci-joint. Les méthodes suivantes ont été utilisées :

$-p \mathrm{H}$ : mesuré sur le terrain au $p \mathrm{H}$-mètre électrique ;

(1) Il est à noter que Martini, dans son remarquable travail d'ensemble sur les Culicidx, paru pourtant la même année que la note relative à Anopheles algeriensis, ne fait aucune allusion à celle-ci et mentionne simplement, comme répartition géographique : Afrique du Nord, Italie, Sardaigne.

(2) Dans ce travail, An. algeriensis, sans indication de localité ni de conditions biologiques, est simplement cité dans un tableau faisant état des stades rencontrés en fonction de la période de l'année. 
- alcalimétrie : dosage en présence d'hélianthine; résultats exprimés en centimètres cubes de $\mathrm{SO}_{4} \mathrm{H}_{2} \mathrm{~N} / 1$ par litre (3) ;

— oxygène dissous : dosé (en surface) par la méthode de Winkler avec l'appareil de Nicloux, exprimé en milligrammes par litre ;

- matières organiques: dosées par oxydation permanganique en milieu alcalin, exprimées en milligrammes par litre :

- ammoniac : recherche au réactif de Nessler ; appréciation de l'intensité de la réaction de 0 (absence) à $5+$ (réaction maximum) (Tr. : traces) ;

- nitrites : recherche aux réactifs de Griess ;

- chlorures : dosés par la méthode de Votocek, exprimés en milligrammes de CINa par litre ;

- calcium et magnésium : dosés au Complexon III en présence de murexide et de noir d'ériochrome, exprimés en milligrammes par litre ;

— degré hydrotimétrique : mesuré au Complexon III et exprimé en degrés français.

Autres caractères: Nous avons noté l'étendue, la profondeur, l'importance de la végétation (dressée, flottante et immergée) et le degré d'ensoleillement (4), à l'endroit précis où les larves étaient récoltées. Chaque collection d'eau de quelque importance constitue en effet souvent un ensemble de biotopes parfois fort différents. Pour plusieurs de ces caractères «indosables», nous avons attribué un coefficient allant de 0 à $5+$. Il est évident qu'une telle attribution de coefficient, n'ayant rien de mathématique et dépendant entièrement de l'appréciation de l'observateur, ne saurait avoir la rigueur d'un dosage. Toutefois, elle garde une valeur certaine lorsque c'est toujours le même observateur qui fait le relevé.

Dans ce tableau, les stades larvaires sont représentés par les chiffres $1,2,3$ et 4 , le stade nymphal par $\mathrm{N}$. La fréquence est indiquée par un nombre de + allant de 1 à 5 . Le stade le plus abondant est souligné.

(3) Il est à noter qu'il n'y a pas parallélisme entre le chiffre du $p H$ et celui du dosage alcalimétrique. Un chiffre élevé pour ce dernier peut avoir en effet pour origine, soit une grande alcalinité vraie, soit un pouvoir tampon élevé.

(4) L'ensoleillement est apprécié au niveau mème de la surface de l'eau. Ainsi, une collection d'eau pourtant largement exposée au soleil, mais très encombrée de végétation dressée, même peu haute, aura un coefficient bas. 


\section{Commentaires}

L'abondance de Th. litorea sur une bonne partie du littoral atlantique français (il est vraisemblable qu'on le trouvera encore plus au Nord quand on le recherchera avec soin) montre bien que cette espèce, loin d'être purement méditerranéenne, a, en réalité, une aire de répartition nettement plus septentrionale que précédemment estimée. Sénevet et Andarelli (1956), en raison de sa rareté en Afrique du Nord, considèrent d'ailleurs que cette espèce atteint là sa limite biogéographique Sud. Il convient donc de la définir comme une espèce européenne des régions tempérées occidentales. L'idée d'une espèce littorale, émise lors de ses premières captures, semble bien se confirmer. En effet, si certains, en Angleterre notamment, et nous-mêmes, l'avons parfois trouvée à l'intérieur des terres, la distance à la mer n'est qu'exceptionnellement considérable (maximum de $15 \mathrm{~km}$. en ce qui concerne nos propres prospections). Et, pourtant, nous prospectons au moins autant les régions de l'intérieur que les zones littorales.

Le nombre des gites que nous étudions ici est évidemment fort peu important (16). Toutefois, l'étude écologique comparée de nombreux gites larvaires hébergeant les deux autres espèces françaises de Culicella et le relevé des associations larvaires observées dans les gîtes de $T h$. litorea permettent d'établir les distinctions suivantes :

\section{Eloignement de la mer:}

Th. litorea, abondant relativement sur le littoral, se raréfie rapidement en pénétrant à l'intérieur des terres.

Th. morsitans, au contraire, rare sur le littoral, devient abondant à l'intérieur.

Th. fumipennis, aussi abondant sur le littoral qu'à l'intérieur.

\section{Richesse en végétation:}

Th. litorea vit dans des collections d'eau riches en végétation surtout dressée (Juncus, Scirpus, Carex, Phragmites et Typha). Nos observations confirment donc celles de Rioux effectuées dans le bassin méditerranéen : « ...rencontré dans des (gìtes) dont la végétation verticale, toujours très dense... » (1958). 
Th. morsitans, au contraire, se rencontre dans des gites variés, souvent pauvres en végétation, mais riches en débris végétaux, feuilles mortes notamment (cette espèce est d'ailleurs très fréquemment associée aux Aedes forestiers).

Th. fumipennis s'observe dans des collections d'eau caractérisées par une richesse extrême en végétation immergée et flottante, mais pauvres en végétation dressée.

\section{Types de gîtes:}

$T h$. litorea se rencontre dans des dépressions de terrain souvent très étendues, à surface largement ouverte. Ce caractère des gîtes a d'ailleurs été antérieurement relevé par Marshall et Staley (1933) : «in open situations ».

Th. morsitans vit dans des collections d'eau souvent petites, fortement ombragées, dans les fossés et les sous-bois.

Th. fumipennis s'observe dans des mares de pâture et dépressions inondées très ensoleillées.

La présence dans des gîtes larvaires souvent proches du littoral implique, chez les larves, une certaine tolérance au chlorure de sodium. Ainsi, comme cela a été antérieurement noté par Marshall et Staley (1933) (salinité pouvant atteindre $15 \%$, soit environ $5 \mathrm{~g}$. de $\mathrm{ClNa}$ /litre) et par Rioux (3,600 g./l.), Theobaldia litorea supporte une concentration relativement élevée. Nous l'avons trouvé dans des eaux de teneur dépassant $5 \mathrm{~g}$./ l. La présence de chlorure de sodium ne semble pas un facteur déterminant, puisque nous l'avons également récolté dans des eaux pauvres en sel (5 gìtes sur 16 avec de 50 à $100 \mathrm{mg}$./l.).

Comme dans le bassin méditerranéen, Theobaldia litorea ne présente qu'une seule génération par an. Les œufs, qui semblent déposés à la fin de l'été par les femelles (peut-être y a-t-il là, comme pour les autres Culicella, un phénomène de véritable diapause estivale), donnent, soit en automne, soit à la fin de l'hiver, des larves qui atteignent l'état de nymphes et donnent des adultes pendant le mois d'avril et le début du mois de mai.

Anopheles algeriensis est nettement moins abondant que Theobaldia litorea sur la côte Ouest. Cependant, nous l'avons trouvé en abondance à l'état larvaire en Charente-Maritime. Par ailleurs, plusieurs larves et de nombreux adultes (ex-nympha) ont été déterminés par Hedeen en Charente-Maritime et en Loire-Atlantique. Enfin, c'est à plusieurs reprises que l'espèce a été signalée 
d'Angleterre. Or, dans la partie française du bassin méditerranéen, deux seuls points de capture sont connus, l'un en Provence (Sautet, 1953), l'autre dans l'Aude (Fontes Tramer, Sicart, 1956). Le premier était si peu important qu'il fut considéré comme une station d'avenir incertain (5). Le second gîte n'a fourni que deux larves. Or, si l'on considère que la côte méditerranéenne et son arrièrepays sont l'objet, depuis de nombreuses années, d'incessantes et encore actuelles prospections culicidologiques (c'est, de très loin, la région de France la plus «fouillée » en ce domaine), que, par opposition, la côte Ouest n'a été que très exceptionnellement l'objet de prospections (mises à part les nôtres, de caractère récent d'ailleurs), on est amené à se demander quelles stations, atlantiques ou méditerranéennes (du moins celles de France), doivent être considérées comme aberrantes. Comme pour Th. litorea, nous sommes persuadés que des recherches plus étendues sur toute la côte Ouest permettront de retrouver ailleurs cette intéressante espèce.

A propos de son écologie larvaire, les renseignements que nous possédons (trois gîtes seulement) sont très restreints. Nous pouvons cependant constater que les trois stations précitées étaient constituées de collections d'eau très encombrées de végétation dressée (Phragmites, Typha ou Juncus), à eau légèrement ou moyennement

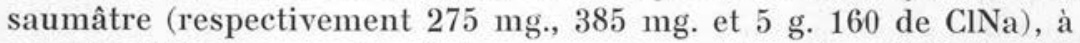
proximité de la mer. Il est à remarquer que l'espèce était chaque fois associée à $T h$. litorea.

C'est d'ailleurs dans des gîtes larvaires de ce genre que Sicart, puis Hedeen l'ont observée ; pour le premier : eau légèrement saumâtre (228 mg. de CINa/l.), très encombrée de végétation dressée ; pour le second, à La Rochelle, dans une mare semi-permanente avec végétation dense. Les renseignements concernant les stations des environs de St-Nazaire manquent malheureusement, les adultes déterminés ayant été obtenus ex-larva de provenances diverses non précisées. Toutefois, aux portes de St-Nazaire, s'ouvre une vaste zone marécageuse très riche en végétation dressée et de plus de cent kilomètres carrés, la Grande-Brière, où Th. litorea est particulièrement abondant. Il est logique de penser que c'est là l'origine des An. algeriensis déterminés par Hedeen.

Le caractère nettement agressif pour l'homme de cette espèce,

(5) C'est en réalité en 1944 que cette station fut observée, à proximité d'un aérodrome servant à un intense trafic aérien avec l'Afrique du Nord, au moment du débarquement (communication personnelle de M. le Professeur Sautet). Ce sont sans doute ces circonstances qui ont amené à considérer la présence d'An. algeriensis sur le soì français comme d'implantation récente au maintien incertain. 
signalé par de nombreux auteurs et vérifié par nous-mêmes, nous fait penser que, si son rôle est sans doute restreint dans certaines régions où existent en abondance des vecteurs reconnus du paludisme, par contre, dans d'autres régions, il faut peut-être tenir compte de sa présence en tant que vecteur possible. Sicart pose d'ailleurs le problème de son rôle éventuel dans la transmission du paludisme dans le Roussillon (6).

\section{Bibliographie}

Artren (T. H. G.), 1955. - The Culicide of Sardinia and Corsica (Diptera). Bull. Entom. Res., 45, p. 437.

Brumpt (E.), 1925. - Les Anophèles de Corse. Bull. Acad. Méd. Paris, 94, p. 811.

Catanei (A.), 1925. - Anophèles des hautes régions de la Corse. Présence d'A. bifurcatus L. et d'A. plumbeus H. et S. dans l'ile. Bull. Soc. Path. exot., 18 , p. 655 .

Dову (J.-M.), 1955. - Localités nouvelles pour quelques espèces de culicidés rarement signalés en France : Uranotænia unguiculata Edwards 1913, Culex impudicus Ficalbi 1890, Culex mimeticus Noé 1899, Theobaldia litorea Shute 1928 et Aedes longitubus Cambournac 1938. Ann. Parasit. hum. et comp., 30, p. 136.

Doby (J.-M.), David (F.) et Rault (B.), 1958. - Contribution à l'étude faunistique et écologique des Culicidés et Simuliidés (Diptera Nematocera) de Belle-Ile (Morbihan). Cahier des Naturalistes, Bull. N.P., n. s., 1/, p. 94. Edwards (F. W.), 1932. - Anopheles algeriensis Theobald (Diptera, Culicida) in Norfolk. Journ. Entom. Soc. England, 1, p. 26.

Harant (H.) et Rioex (J. A.), 1954. - Theobaldia (Culicella) litorea (Shute), Culicidé nouveau pour la France. Cahier des Naturalistes, Bull. N.P., n. s., 9, p. 57.

Hant (G. F. W.), 1954. - Rediscovery of Anopheles algeriensis Theo. (Dipt., Culicida) in Norfolk. Entom. Mon. Mag., n 171, p. 63.

HEDEEN (R. A.), 1957. - A note on the occurrence of Anopheles algeriensis Theobald in Western France. Mosquito News, 17, p. 97.

Jaujou (C.), Camaix (R.) et Adam (J.-P.), 1950. - Plan de mise en valeur de la Corse. Rapport sur l'activité du service antipaludique en 1919. Ajaccio, Impr. Siciliano.

Jaujou (C.), Michel (L.) et Hamon (J.), 1951. — Plan de mise en valeur de la Corse. Rapport sur l'activité du service antipaludique en 1950. Paris, Alepée et $\mathrm{C}^{\text {ie }}$ édit.

(6) En Charente-Maritime, en dépit de la grande abondance d'Anopheles maculipennis (var. atroparvus), nous n'avons jamais été attaçués par cette cspèce. Or, il est courant, dans cette région, d'entendre parler de « fièvre de marais », et un médecin, ancien colonial, y exerçant depuis de nombreuses années, nous a affirmé récemment que les cas de paludisme chez des autochtones n'ayant jamais quitté le pays, cas d'ailleurs souvent cliniquement atypiques, mais confirmés par la mise en évidence du parasite (Pl. vivax) dans le sang, n'étaient pas chose extraordinaire. Si de tels faits étaient confirmés, il faudrait alors penser que le paludisme n'a peut-être pas régressé dans cette région autant qu'il est classique de le dire et que e'est éventuellement dans la présence d'Anopheles algeriensis qu'il faut en chercher la raison. 


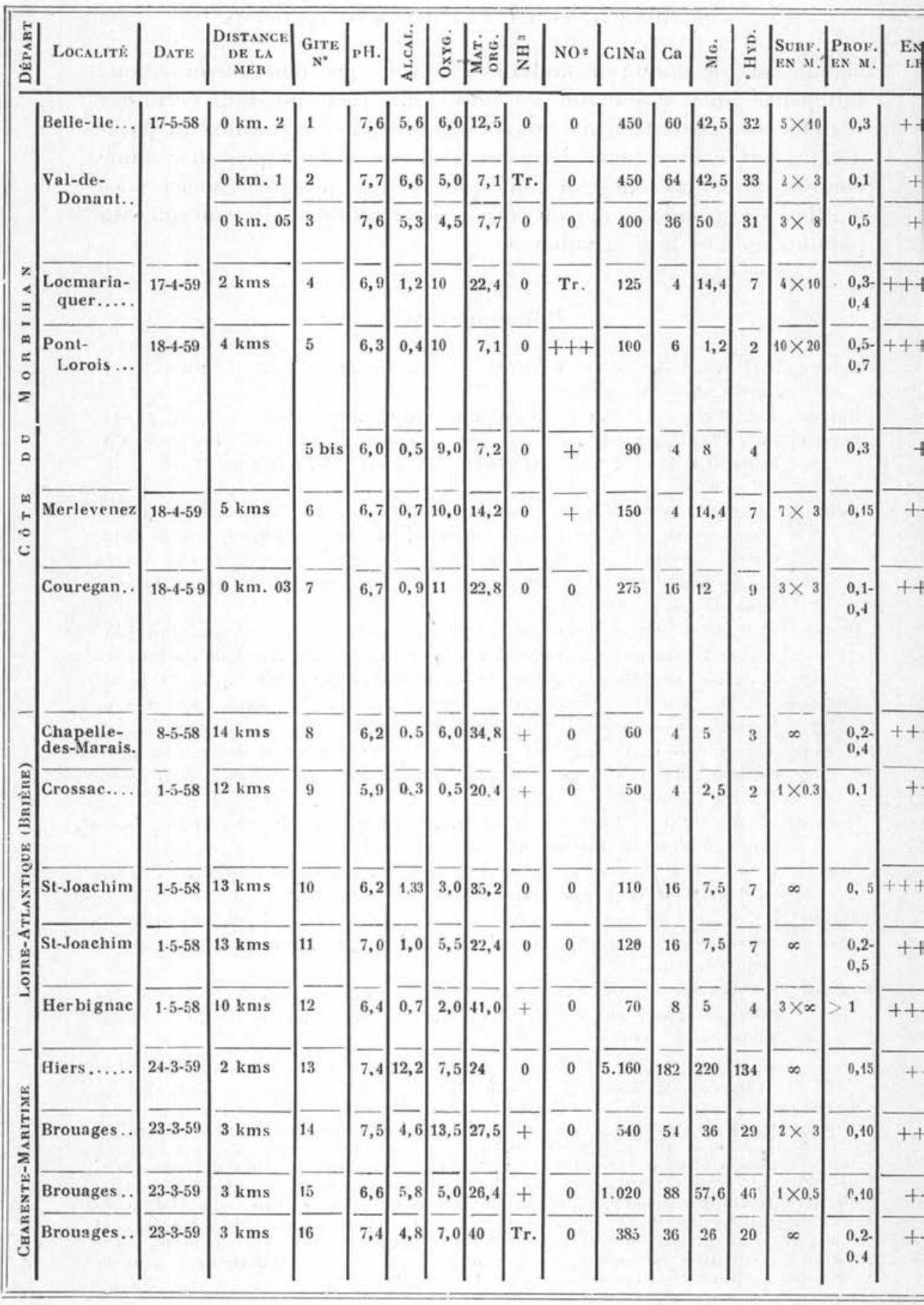




\begin{tabular}{|c|c|c|c|c|c|c|c|}
\hline \multirow{2}{*}{$\begin{array}{l}\text { us } \\
\text { Aux }\end{array}$} & \multicolumn{3}{|c|}{ VÉGETATION } & \multirow{2}{*}{ Algues } & \multirow{2}{*}{ TYPE DE GITE } & \multirow{2}{*}{$\left|\begin{array}{c}\text { STADES DE } \\
\text { Theobaldia } \\
\text { litorea }\end{array}\right|$} & \multirow{2}{*}{ ESPÈCES ASSOCIÉES } \\
\hline & Dressè । & Flot. I & | Immer. & & & & \\
\hline+ & +++ & ++ & +++++ & $\underset{\text { brunes }}{+++}$ & Dépression inondée & N. $t+t$ & Th. fumipennis $\mathrm{N} .+$ \\
\hline+ & ++++ & + & $+++t$ & 0 & Dépression inondée & 4. N. +++ & Th. fumipennis 4. + \\
\hline+ & $+++t$ & ++ & ++ & 0 & $\begin{array}{c}\text { Mare libre avec bords } \\
\text { encombrés de phragmites }\end{array}$ & N. ++ & Th. fumipennis N. + \\
\hline+ & +++ & ++ & $+t+t$ & 0 & Zone marécageuse & N. + & $\begin{array}{l}\text { Th. fuminpenis } \mathrm{N} .++ \\
\text { An. maculipennis } 4 .++\end{array}$ \\
\hline+ & $+t$ & ++ & +++ & 0 & $\begin{array}{c}\text { Grande mare(prélèvement } \\
\text { à une extrémité riche } \\
\text { surtout en végétation } \\
\text { immergée) }\end{array}$ & N. + & $\begin{array}{l}\text { Th. fumipennis } 4 . \text { N. }+++ \\
\text { dn. maculipennis } 3.4 .++\end{array}$ \\
\hline+ & ++++ & 0 & $+t+$ & 0 & $\begin{array}{l}\text { Id. (extrémité nvec louffes } \\
\text { serrées de juncus) }\end{array}$ & 4. $\mathrm{N}+++$ & Th. fumipennis 4. $\mathrm{N}+$ \\
\hline $\begin{array}{c}-++ \\
\text { illes } \\
\text { tes) }\end{array}$ & ++ & 0 & ++ & 0 & $\begin{array}{c}\text { Mare } \\
\text { (extrémité sous buissons) }\end{array}$ & 4. ++ & $\begin{array}{l}\text { Th. fumipennis } 3.4 .+ \\
\text { Th. morsitans } 4 .++\end{array}$ \\
\hline++ & $+t+t$ & + & ++++ & 0 & $\begin{array}{l}\text { Dépression dans dunes } \\
\text { encombrée de juncus }\end{array}$ & N. +++ & $\begin{array}{l}\text { Th. fumipennis } \mathrm{N} .++ \\
\text { An. algeriensis } \mathrm{N} .(1) \\
\text { An. maculipennis } 2 .+ \\
\text { Cul. pipiens } 1 .+ \\
\text { Aed. detritus } 2.3 .++ \\
\text {.ed. caspius } 1 .++\end{array}$ \\
\hline+ & ++ & ++ & ++++ & + brunes & Vaste dèpression inondée & N. + & Th. fumipennis N. ++ \\
\hline $\begin{array}{l}+ \\
\text { illes } \\
\text {-tes) }\end{array}$ & +++ & 0 & 0 & 0 & Petit fossé sous haies & N. ++ & $\begin{array}{l}\text { Th. funipennis } 4 . \mathrm{N} .+ \\
\text { Th. morsitans N. + } \\
\text { Mochlonyx culiciformis N. } \\
\quad+\end{array}$ \\
\hline 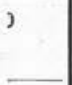 & $+t$ & ++ & +++++ & 0 & Large dépression inondée & $\mathrm{N}+$ & $\begin{array}{l}\text { An. maculipennis } 3 .++ \\
\text { Th. fumipennis 4. N. }+ \\
\end{array}$ \\
\hline+ & +++ & + & +++++ & 0 & Large dépression inondée & 4. N. ++ & $\begin{array}{l}\text { Th. fumipennis } 4 . \mathrm{N}+{ }_{+}^{+} \\
\text {An. maculipennis } 4 .+\end{array}$ \\
\hline+ & ++ & 0 & ++ & 0 & $\begin{array}{c}\text { Canal avec végétation } \\
\text { en touffes }\end{array}$ & N. + & Th. fumipennis N. ++ \\
\hline+++ & +++++ & + & +++ & 0 & $\begin{array}{l}\text { Zone marécageuse } \\
\text { a phragmites }\end{array}$ & 4. N. + & An. algeriensis $4 . \mathrm{N}$. \\
\hline+ & ++ & +++ & +++ & 0 & $\begin{array}{c}\text { Dépression marécageuse } \\
\text { dans pâture }\end{array}$ & N. + & $\begin{array}{l}\text { An. maculipennis } 1.2 .+ \\
\text { Th. fumipennis } 4 . \mathrm{N} .+++ \\
\end{array}$ \\
\hline+ & +++++ & ++ & +++ & 0 & Dépression de drainage & 4. ++ & Th. fumipennis $4 .++$ \\
\hline+ & +++++ & ++++ & +++ & 0 & Marais à $T y p h a$ & 4. N. $+t$ & $\begin{array}{l}\text { An. algeriensis 3. } 4 .++ \\
\text { An. maculipennis } 1 .+ \\
\text { Chaoborus sp. } 3 \text { 4. N. }+++\end{array}$ \\
\hline
\end{tabular}


Macan (T. T.), 1933. - An inland record for Theobaldia (Culicella) litorea. Entomologist, 66, p. 261.

Macan (T. T.), 1939. - The Culicide of the Cambridge District. Parasitology, 31. p. 263.

Marshal. (J. F.), 1938. - The British Mosquitoes. British Museum of Natural History, Londres.

Marshall (J. F.) et Staley (J.), 1933. - Theobaldia (Culicella) litorea (Shute) n. sp. (Diptera, Culicide). Parasitology, 25, p. 119.

Mattingly (P. F.). - in Senevet et Andarelli, 1956.

MARtini (E.), 1931. - Ueber Anopheles bifurcatus und algeriensis. Anz. Schladingsk, Berlin, 7, p. 109.

Martini (E.), 1931. - Culicide. In Lindner. Palaaretic Fliegen. Stuttgart.

Rıoux (J.-A.), 1958. - Les Culicides du «Midi» méditerranéen. Lechevalier éd., Encyclopédie entomologique, Paris.

SaUter (J.), 1953. - Les fluctuations du paluc'isme dans le Sud de la France et dans les départements insulaires. Rev. Pathol. génér. et comp., $\mathrm{n}^{\circ} 652$, p. 1199.

Senevet (G.), 1935. - Les Anophèles de la France et de ses colonies. I. France, Corse, Afrique, Madagascar, La Réunion. Lechevalier éd., Encyclopédie entomologique, Paris.

Senevet (G.) et Andarelli (L.), 1956. - Les Anophèles de l'Afrique du Nord et du bassin méditerranéen. Lechevalier éd., Paris.

Senevet (G.) et Andarelli (L.), 1956. - Présence en Algérie de Theobaldia litorea (Shute). Archives Inst. Pesteur d'Algérie, 34, p. 400.

Shute (P. G.), 1928. - A new variety of Culicella morsitans (Theobald). Entomologist, 61, p. 186.

ShtTe (P. G.), 1933. - The life history and habits of british mosquitoes in relation to their control by antilarval operations. Jl. of Trop. Med. a. Hyg., 36, p. 83.

Sicant (M.), 1956. - Sur la présence d'Anopheles algeriensis Theobald en Roussillon. Montpellier Médical, 49, p. 480.

ionres-Chñamares (F.), 1945. - Culieidos de la Provincio de Cuenea (Dipt. Cul.). Rev. San. Hig. publ., 19, dẻeembre.

ToumanofF (C.), 1954. - Contribution à l'étude des anophèles de Corse. Inst. Nation. Hygiène, Paris.

(Laboratoire de Zoologie et Parasitologie de la Faculté de Médecine et de Pharmacie de Rennes). 


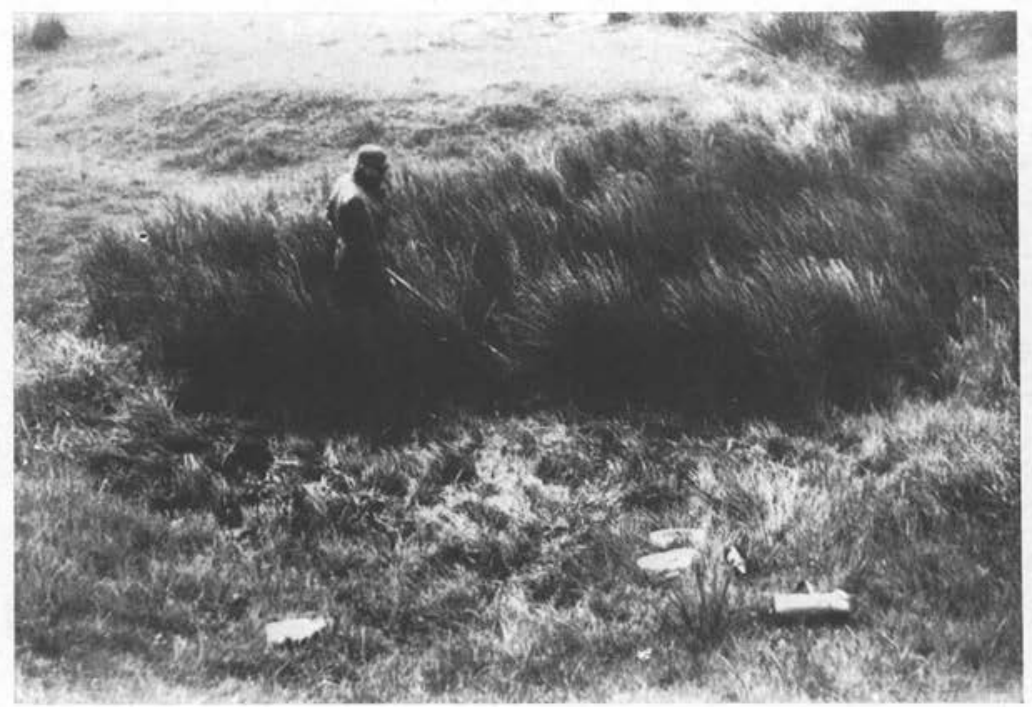

Gîte $n^{\circ}$ 2. - Val de Donant (Belle-Ile-en-Mer). Dépression peu profonde entièrement occupée par Juncus glaucus et Scirpus maritimus. Gîte à Th. litorea.

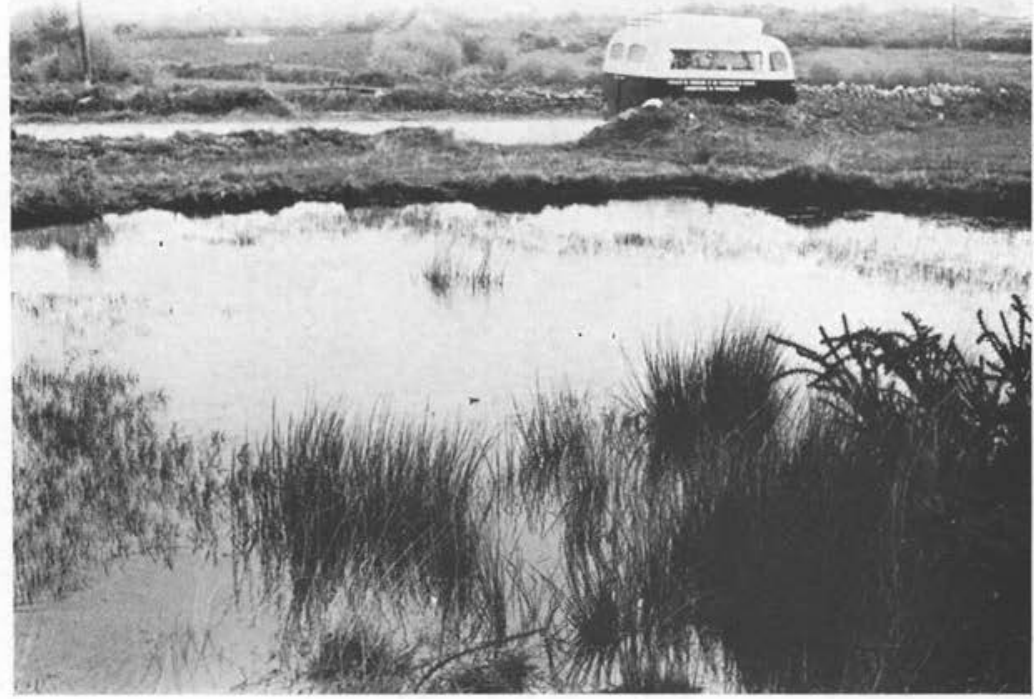

Gîtes $\mathrm{x}^{\circ} 5^{\text {b1s }}$ et 5 . - Pont-Lorois (Morbihan). Au premier plan, zone très encombrée de végétation dressée (Juncus). Nombreux Th. litorea. Rares Th. fumipennis. Au dernier plan, zone avec végétation flottante et immergée. Nombreux Th. fumipennis et An. maculipennis. Très rares Th. litorea.

ANs. de Parasitologie, T. XXXV, $\mathrm{N}^{\circ} 1-2 .-1960$. 


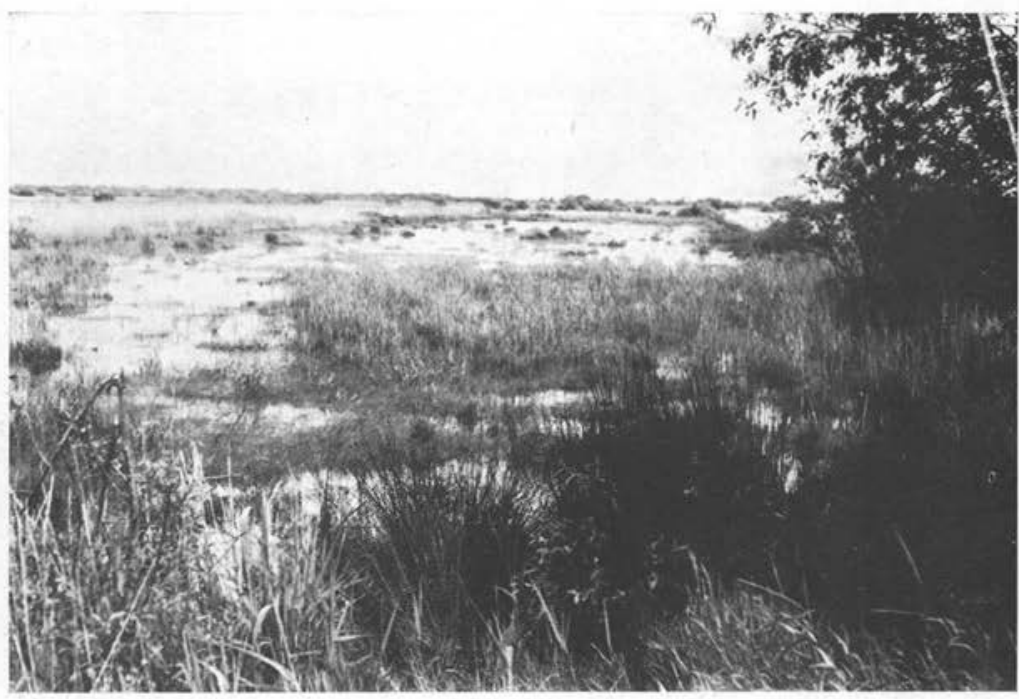

Gîte $n^{\circ}$ 8. - La Chapelle-des-Marais (Grande-Brière, Loire-Atlantique). Marais à Phragmites communis, Carex acuta et Juncus heterophyllus. Gite à Th. lito. rea et Th. fumipennis.

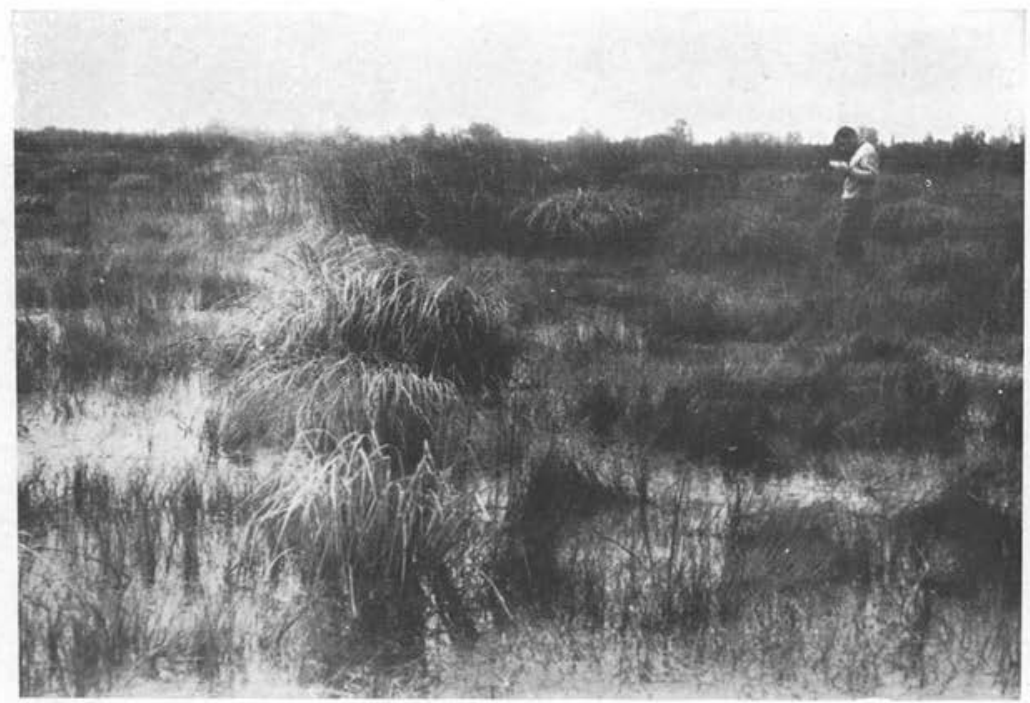

Gîte ${ }^{\circ}$ 11. - St-Joachim (Grande-Brière, Loire-Atlantique). Marais avec grosses touffes de Scirpus lacustris et Carex acuta (Th. litorea). Végétation flottante et immergée de Scirpus fuitans, Juncus heterophyllus et Hydrocotyle vulgaris (Th. fumipennis et An. maculipennis). 


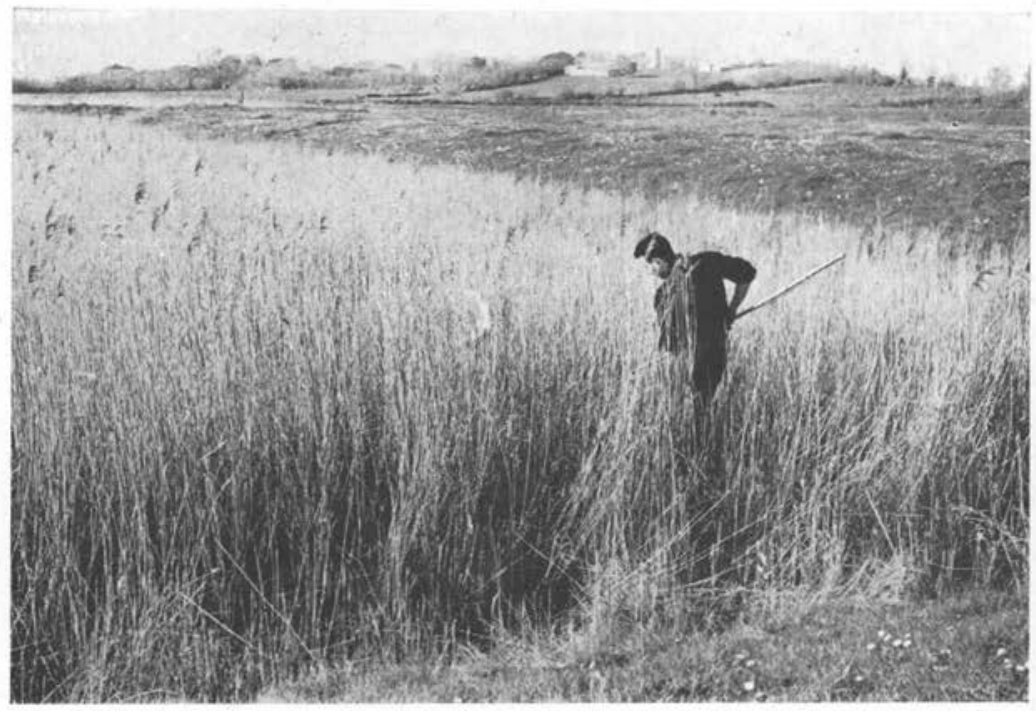

Gîte $n^{\circ}$ 13. - Hiers (Charente-Maritime). Grandes dépressions entièrement couvertes de Phragmites. Gîtes à An, algeriensis et à $T h$. litorea.

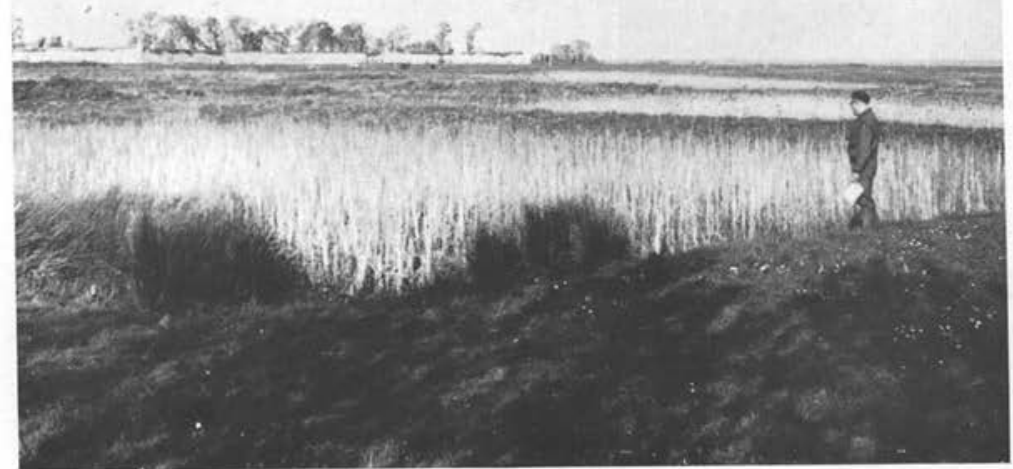

Gìte $\mathrm{n}^{\circ}$ 16. - Brouages (Charente-Miaritime). Grandes dépressions bordées de Juncus, très encombrées de Typha (quelques Phragmites). Gîtes à An. algeriensis, An. maculipennis et Th, litorea (dans le fond, les fortifications de Brouages). 